Conclusion: The results from this exploratory study in a large cohort of SSc patients provide valuable insights into factors associated with digital pitting. Patients with digital pitting often have an earlier onset of Raynauds and of SSc and significantly more debridements/amputations, suggesting that digital pitting is associated with vascular disease severity. Our findings indicate the need for further research investigating pathophysiology of digital pitting, to inform development of preventative treatment strategies.

Disclosure of Interests: None declared

DOI: 10.1136/annrheumdis-2020-eular.1028

\section{AB0623 RATE AND PREDICTIVE FACTORS ASSOCIATED WITH SUSTAINED REMISSION IN IDIOPATHIC INFLAMMATORY MYOSITIS}

T. Vojinovic ${ }^{1}$, X. Gjoni ${ }^{2}$, M. Fredi ${ }^{1}$, F. Franceschini ${ }^{1}$, I. Cavazzana ${ }^{1} .{ }^{1}$ ASST Spedali Civili, Rheumatology and Clinical Immunology Unit, Brescia, Italy; ${ }^{2}$ University of Brescia, Brescia, Italy

Background: Idiopathic inflammatory myositis (IIM) is a group of heterogeneous connective tissue diseases, characterised by chronic muscle inflammation, myositis-specific or myositis-associated autoantibodies and different extra-muscular features. Achieveing low disease activity or remission in patients with IIM has proven to be difficult due to the wide clinical spectrum of the different IIM types.

Objectives: To retrospectively assess any predictive factors for sustained remission in IIM patients

Methods: We retrospectively analyzed data taken from medical charts, which included age at disease onset, gender, laboratory data as well as clinical features present at onset, organ involvement and treatment history. A total of 151 adult patients with IIM followed-up for > 1 year were retrospectively enrolled. Remission was defined as no clinical and laboratory evidence of disease activity persistent for more than 6 months during follow-up, while undergoing myositis therapy or under no medication. The remission of cutaneous involvement was defined as no current activity of skin rash, absence of Gottron's papules as well as heliotrope rash and erythema, whereas the remission of pulmonary involvement was considered as no requirement for intensification of immunosuppressive therapy during follow-up. Likewise, absence of muscle weakness or hyposthenia was taken into account for evaluating muscle involvement. Moreover, the clinical features were accompanied by normalization of myogenic enzymes such as creatine kinase $(\mathrm{CK})$ and lactate dehydrogenase levels.

Results: Among all 151 patients, 89 (58.9\%) patients achieved sustained remission. By univariate analysis, overlap myositis (7.9\% vs $27.4 \% ; p=0.003$; OR:0.22), cancer-associated myositis (CAM) (7.8\% vs $19.35 \%$; $\mathrm{p}=0.046$; OR:0.3), as well as the presence of anti $\mathrm{Ku}(3.37 \%$ vs $12.9 \% ; \mathrm{p}=0.05$; OR:0.23) and anti TIF-1 gamma ( $1.1 \%$ vs $8 \% ; p=0.043$; OR:0.13) antibodies and polyarthritis ( $11.2 \%$ vs $24.19 \% ; p=0.045$; OR: 0.397 ) at onset were significantly associated with active IIM, not achieving remission.

Out of 89 patients in remission, 79 (88.8\%) achieved long-term sustained remission, lasting at least 2 years, whereas 10 patients (11.2\%) showed relapse. Most of relapsed patients showed a relapse/remitting disease, with no evident trigger for the relapse. We divided our cohort of 89 patients in remission in 2 subsets: 83 patients in remission undergoing therapy (93.3\%) and 6 patients in drug-free remission $(6.7 \%)$. No significant differences were found between two groups, except for younger age at onset $(p=0.0002)$ found in patients achieving drug-free remission.

Conclusion: Sustained remission occurs in about one half of patients with IIM. The presence of anti Ku and anti TIF-1 gamma antibodies as well as polyarthritis at onset lowers the chance of achieving sustained remission. Younger age at diagnosis has proved to predict drug-free long-lasting remission.

Disclosure of Interests: None declared

DOI: 10.1136/annrheumdis-2020-eular.2677

\section{AB0624 \\ PREDICTIVE PARAMETERS FOR DEVELOPMENT OF INTERSTITIAL LUNG DISEASE IN IDIOPATHIC INFLAMMATORY MYOSITIS}

T. Vojinovic $^{1}$, M. Fredi2, P. Ceruti ${ }^{3}$, D. Modina ${ }^{3}$, F. Franceschini2, I. Cavazzana2. ${ }^{1}$ ASST Spedali Civili, Rheumatology and Clinical Immunology Unit, Brescia, Italy; ${ }^{1}$ ASST Spedali Civili, Rheumatology and Clinical Immunology Unit, Brescia, Italy; ${ }^{3}$ ASST Spedali Civili, Pneumology Unit, Brescia, Italy
Background: Idiopathic Inflammatory Myositis (IIM) is a group of heterogeneous connective tissue diseases, primarily characterized by chronic muscle inflammation as well as myositis-specific or myositis-associated autoantibodies and a spectrum of different extra-muscular features.

The most frequent organ involment in IIM is Interstitial Lung Disease (ILD) occurring in $5-80 \%$ of different IIMs cases and considered the hallmark of morbidity and mortality in patients with IIMs.

Objectives: To retrospectively assess the predictive factors for development of ILD in IIM patients

Methods: We retrospectively analyzed the prevalence of ILD in a single-center cohort of 165 IIM patients. Patient data was collected from clinical charts. ILD was diagnosed by chest X-ray scan and chest CT scan. Al chest $\mathrm{CT}$ and chest $\mathrm{X}$-ray scans available and performed at our hospita were consequently re-evaluated by our expert pneumologist for uniform evaluation.

Results: Myositis-related ILD (M-ILD) was found in 52 IIM patients (31.5\%) $46.15 \%$ was affected by anti-synthetase syndrome (ARS), $21.15 \%$ by polymyositis (PM), $19.23 \%$ by dermatomyositis (DM) and $13.46 \%$ by overlap myositis. The pulmonary involvement was characterized by Non-specific interstitia pneumonia (NSIP) (30.6\%), Unusual Interstitial Pneumonia (UIP) (38.77\%) Bronchiolitis Obliterans with Organizing Pneumonia (BOOP) $(20.4 \%)$, overlap NSIP/BOOP (4.1\%) and Undetermined/Unspecific pattern (6.12\%). Eighty four percent of M-ILD consisted of non-smokers and $69.23 \%$ presented with dyspnea at onset.

ILD was diagnosed in $90.38 \%$ of patients within the first year of IIM diagnosis (early onset ILD) and was associated with dyspnea and/or cough in $70.2 \%$ and $17 \%$ respectively. On the other hand, late onset ILD presented mostly with dyspnea and/or cough in $60 \%$ of cases and was significantly associated with anti-Ku antobodies.

At onset ILD was significantly associated with: ARS ( $p<0.0001 ; 0 R: 12.98)$ anti-Jo-1 ( $p<0.0001$; OR:6.1), anti-Ro $(p=0.038$; OR:2.2), mechanic's hands $(p<0.0001$; OR:10.41), arthritis $(p=0.01$; OR:2.58), polyarthritis ( $p=0.001$; OR:4.578), dyspnea $(p<0.0001$; OR:9.66), and high levels of CPK $(p=0.0001)$ and GOT $(p=0.0146)$. By contrast, the following features: $D M(p=0.012 ;$ OR:0.36), facial rash $(p=0.003 ;$ OR:0.31), anti-NXP-2 $(p=0.019 ; \quad O R<0.0001)$, anti-PL-12 $(p=0,03 ; O R<0.0001)$ and myositis $(p<0.0001$; OR:0.173) present at onset were less frequently associated with M-ILD.

At multivariate analysis M-ILD was predicted by anti-Ro $(p=0.0448)$, polyarthritis $(p=0.0093)$ and dyspnea $(p=0.0001)$ at onset. On the other hand, patients presenting myositis $(p=0.0383)$ and facial rash $(p=0.0398)$ at onset were less likely to developed M-ILD.

Conclusion: ILD occurs in about one third of patients with IIM, mostly affected by ARS. The presence of anti-Ro antibodies as well as polyarthritis and dyspnea at onset predict the development of ILD.

Disclosure of Interests: None declared

DOI: 10.1136/annrheumdis-2020-eular.3571

\section{AB0625 IS PULMONARY ARTERIAL HYPERTENSION, ASSOCIATED WITH SYSTEMIC SCLEROSIS REVERSIBLE?}

A. Volkov ${ }^{1}$, N. Yudkina ${ }^{1}$, E. Nikolaeva ${ }^{1}$, M. Starovoytova ${ }^{1}$, O. Alekseeva ${ }^{1}$ I. Nikishina ${ }^{1}$, E. Nasonov ${ }^{1}{ }^{1}$ VA Nasonova Research Institute of Rheumatology, Moscow, Russian Federation

Background: Systemic sclerosis (SSc) is one of the frequent causes of the pulmonary arterial hypertension (PAH) which found in $6-15 \%$ patient with SSc Patients with $\mathrm{PAH}$, associated with $\mathrm{SSc}$ have a poorer prognosis than other forms of $\mathrm{PAH}$. 6 World Symposium of $\mathrm{PH}$ lowered diagnostic cut-off to $21 \mathrm{~mm} \mathrm{Hg}$ in hope of improved survival. $\mathrm{PAH}$ reversibility is described in congenital heart defects, HIV and some tumors

Objectives: The aim of the study to detect reversibility of PAH associated with SSc.

Methods: Hemodynamics (mean pulmonary artery pressure (MPAP), pulmonary artery wedge pressure (PAWP), cardiac output $(\mathrm{CO})$ and pulmonary vascular resistance (PVR)), functional class (NYHA), 6-minute walk distance (6MWD), biomarkers and DLCO were assessed. Patients with pulmonary fibrosis and left heart diseases were excluded.

Results: The study includes 56 patients receiving start-up monotherapy with 1st generation PAH-specific drugs: bosentan (25 patients) and silde nafil (31 patients). The median age of the patients was $51.5(37 ; 58)$ years. At the time of diagnosis, the MPAP in the sildenafil group was $49(30$ 50) $\mathrm{mm} \mathrm{Hg}$, bosentan - $50(42 ; 56) \mathrm{mm} \mathrm{Hg}$, differences not significant $(p=0.11)$. During observation against the background of sildenafil intake, 